\title{
Determinación De La Influencia De Las Fases \\ Lunares, Utilizando Tres Variedades De Fréjol (Phaseolus Vulgaris L.) En El Cantón Antonio Ante, Provincia De Imbabura
}

\author{
Ing. Agro. Maribel Vera Suárez \\ Ing. Agro. Ricardo Chávez Batancourt \\ Ing. Agrop. Víctor Molina \\ Ing. Agro. Joffre León Paredes \\ Ing. Agro. Orlando Olvera Contreras \\ Mvz. Hugo Alvarado Álvarez. \\ Ing. Agro. Luis Lahuasi Guerrero \\ Universidad Técnica de Babahoyo
}

doi: 10.19044/esj.2017.v13n6p190 ～URL:http://dx.doi.org/10.19044/esj.2017.v13n6p190

\begin{abstract}
The present study was conducted at the parish of San Roque, canton Antonio which is before the county of Imbabura. The seed materials used in this study are seeds of frijol varieties such as Paragachi, Canario, and Yunguilla which comes from Santa Catalina (INIAP). The treatments were made up of fréjol varieties (Phaseolus vulgaris $L$ ) through the use of the lunar calendar. It uses the design blocks totally at random (DBCA) with six treatments and four repetitions. Their variables were subjected to the variance analysis and it was used to determine the statistical differences of multiple ranges. The Tukey test was also used in this study. The development of the experimental rehearsal were carried out by the following works based on the analysis of the floor, preparation of the floor, subscriber, sowing, fertilization, watering, control of overgrowths, control Phytosanitary, and harvest. To estimate the effects of the treatments, the following data was collected: height from the plant to 30, 60, and 90 days after planting, days of flowering; days of maturation; longitude of sheaths; number of sheaths for plant; number of seeds for sheath; weigh of 100 seeds; grain yield; and economic analysis. According to the interpretation of the results, it was deduced that the biggest plant height was obtained by planting variety of Paragachi. This is done with the use of the lunar calendar. Also, the planting of variety of Yunguilla, using the agricultural lunar calendar, reported bigger number of sheaths for plant and seeds for sheath. The variable days of flowering and days of maturation shows
\end{abstract}


a highly significant difference in the studied treatments. The biggest longitude of sheaths $(14,55 \mathrm{~cm})$; weigh of 100 seeds $(73,25 \mathrm{~g})$, and yield $(3325,00 \mathrm{~kg} / \mathrm{ha})$ presented canario variety using lunar calendar. With regards to the economic analysis, all the treatments obtained profitable results. The canario variety stands out using the agricultural lunar calendar with a bigger net profit of $\$$ 3274,14 .

Keywords: Cocoa, phase of the Moon, seeds, treatments

\section{Resumen}

La presente investigación se efectuó en la parroquia San Roque, cantón Antonio Ante, provincia de Imbabura, utilizando como material se siembra semillas de frejol variedades Paragachi, Canario y Yunguilla, provenientes de Santa Catalina (INIAP). Los tratamientos estuvieron constituidos por las variedades de fréjol (Phaseolus vulgaris L) con la utilización del calendario lunar, empleando el diseño bloques completamente al azar (DBCA) con seis tratamientos y cuatro repeticiones, cuyas variables fueron sometidas al análisis de varianza y para determinar las diferencias estadísticas de rangos múltiples se utilizó la prueba de Tukey. Para el desarrollo del ensayo experimental se realizaron las siguientes labores como análisis del suelo, preparación del suelo, abonado, siembra, fertilización, riego, control de malezas, control fitosanitario y cosecha. Para estimar los efectos de los tratamientos se tomaron los datos como altura de la planta a los 30,60 y 90 días después de la siembra, días a floración; días a maduración; longitud de vainas; número de vainas por planta; número de semillas por vaina; peso de 100 semillas; rendimiento de grano y análisis económico. De acuerdo a la interpretación de los resultados, se deduce que la mayor altura de planta se obtuvo sembrando la variedad Paragachi, con la utilización del calendario lunar; la siembra de la variedad Yunguilla, empleando el calendario lunar agrícola reportó mayor número de vainas por planta y semillas por vaina; las variables días a floración y días a maduración, reportaron diferencias altamente significativas en los tratamientos estudiados. La mayor longitud de vainas $(14,55 \mathrm{~cm})$; peso de 100 semillas $(73,25 \mathrm{~g})$ y rendimiento $(3325,00 \mathrm{~kg} / \mathrm{ha})$ lo presentó la variedad Canario utilizando calendario lunar; respecto al análisis económico, todos los tratamientos obtuvieron resultados rentables, destacándose la variedad Canario, utilizando el calendario lunar agrícola, el que presentó mayor beneficio neto con $\$$ 3274,14 .

Palabras clave: Cacao, fase lunar, semillas, tratamientos 


\section{Introducción}

El fréjol (Phaseolus vulgaris L), es un cultivo típico entre los pequeños productores de América Central y del Sur, y principal fuente proteica para una parte significativa de la población en gran número de zonas en las que la agricultura de subsistencia es la principal actividad productiva. Este cultivo, es entre las leguminosas de grano alimenticio, la especie más importante para el consumo humano, debido a que su valor nutritivo es una alternativa para suplementar el déficit de la dieta humana en la población mundial. El cultivo de fréjol está repartido en todas las zonas agrícolas a nivel mundial, la superficie dedicada a este cultivo abarca unos 27.5 millones de hectáreas, con una producción de 19 millones de toneladas y un rendimiento medio de 680 $\mathrm{kg} / \mathrm{ha}$. La superficie sembrada en la sierra ecuatoriana fue de 8570 has, de fréjol seco, de las cuales se cultivaron 8160 , con un rendimiento de $0.54 \mathrm{t} / \mathrm{ha}$ (Estadísticas Agropecuarias del Ecuador, 2011).

Para los agricultores de la serranía ecuatoriana agrícola el calendario lunar cobra mucha importancia y es su guía, las creencias populares indican que los agricultores siembran cuando la luna se encuentra en la fase de cuarto creciente o menguante, no realizan ninguna tarea en fase de luna llena, ni cosechan en cuarto creciente. El Calendario Agrícola Lunar nace a mediados de 1990 como una iniciativa particular para apoyar la producción agrícola en el Ecuador y posteriormente la producción pecuaria, apícola y acuícola. La fuente de información original son los principios de la agricultura biodinámica con adaptación para la zona tropical. Aunque, originalmente se apuntaba al uso exclusivo para la práctica de la agricultura sin químicos, ya que se argumentaba que con el uso de los químicos el influjo lunar estaba neutralizado. Sin embargo se pudo constatar que esto no era verdad, pues quienes practicaban la agricultura química lograban mejores resultados cuando realizaban sus labores en los días indicados, e incluso llegaban a requerir menor cantidad de insumos químicos para obtener una producción satisfactoria. Ante esta realidad, el Calendario Agrícola tienen una proyección general en las prácticas productivas, tanto si se trata de una agricultura química, orgánica, biológica, ecológica, biodinámica o de otra naturaleza.

Después de 20 años, con una presencia anual de difusión constante de la influencia de los ritmos lunares en el Ecuador, considero de suma importancia realizar esfuerzos y direccionamiento a las fuentes que impulsaron la creación de esta iniciativa o proyecto de difusión para una agricultura más sana. El calendario nace y justifica el uso y abuso que los seres humanos hacen con los insumos químicos, insumos que se constituyen en un vergonzoso envenenamiento al ambiente, sus recursos "suelo, aire, agua" y todos los seres que lo habitan. Sin embargo, en un peldaño superior, se encuentra la práctica de la Agricultura Biodinámica, la misma que integra las variaciones del Cosmos "Sol, Luna, planetas y constelaciones" para elegir 
momentos oportunos para las actividades agropecuarias e integrando diversos preparados llamados biodinámicas que ayudan y mejoran la producción.

La agricultura es una actividad que se desarrolla en íntima relación con la tierra, el agua, el viento, la luz, la flora, la fauna, la Luna, el Sol, el Cosmos y por supuesto su creador, el ser humano. Por lo tanto, todo el conocimiento y las técnicas que se empleen en la agricultura deben abordar, considerar e integrar los factores indicados.

Por tanto, la presente investigación, estudió alternativas para utilizar el calendario agrícola, debido a la influencia de las fases lunares, para mejorar los niveles de productividad y producción en el cultivo de fréjol.

\section{Objetivo General}

Determinar la influencia de las fases lunares, utilizando el calendario agrícola lunar, en tres variedades de fréjol (Phaseolus vulgaris L). En el cantón Antonio Ante, provincia de Imbabura.

\section{Objetivos Específicos}

$>$ Identificar la variedad de mayor producción, considerando la utilización del calendario agrícola lunar.

$>$ Evaluar los efectos de las fases lunares en el cultivo de fréjol.

\section{Materiales y métodos}

\section{Ubicación y Descripción del Área Experimental}

La presente investigación se efectuó en la parroquia San Roque, cantón Antonio Ante provincia de Imbabura.

La zona presenta una altura de $2.240 \mathrm{msnm}$, temperatura promedio anual de $17.6^{\circ} \mathrm{C}$, humedad relativa promedio anual $75 \%$, precipitación media anual entre $500-1000 \mathrm{~mm}$ y heliofanía media anual 1916.9 horas.

El tipo de suelo es franco arcillo-arenoso y la zona de vida es bosque seco Montano Bajo (bs - MB).

\section{Material de Siembra}

Como material se siembra se utilizó semillas de frejol variedades Paragachi, Canario y Yunguilla, provenientes de Santa Catalina (INIAP).

\section{Factores Estudiados}

Variable Independiente: comportamiento agronómico del fréjol $(P$. vulgaris $L$ )

Variable Dependiente: fases lunares, mediante la utilización del calendario agrícola. 


\section{Tratamientos}

Los tratamientos estuvieron constituidos por las variedades de fréjol (Phaseolus vulgaris L) con la utilización del calendario lunar, los mismos que se detallan en el cuadro siguiente.

Cuadro 1. Detalle de tratamientos, en función de variedades de fréjol y calendario agrícola

\begin{tabular}{|c|c|c|}
\hline \multicolumn{3}{|c|}{ Tratamientos } \\
\hline $\mathrm{N}^{\mathbf{0}}$ & Variedades & Calendario Lunar \\
\hline $\mathrm{T} 1$ & Paragachi & Calendario \\
\hline $\mathrm{T} 2$ & Paragachi & Sin calendario \\
\hline $\mathrm{T} 3$ & Canario & Calendario \\
\hline $\mathrm{T} 4$ & Canario & Sin calendario \\
\hline T5 & Yunguilla & Calendario \\
\hline T6 & Yunguilla & Sin calendario \\
\hline
\end{tabular}

\section{Métodos}

Se emplearon métodos de inducción - deducción, análisis - síntesis y método práctico denominado experimental.

\section{Diseño Experimental}

Se utilizó el diseño denominado bloques completamente al azar (DBCA), con seis tratamientos y cuatro repeticiones.

\section{Análisis de Varianza (ADEVA)}

\begin{tabular}{|c|c|}
\hline Fuentes de Variación & GL \\
\hline Total & 23 \\
\hline Tratamientos & 5 \\
\hline Bloques & 3 \\
\hline Error Experimental & 15 \\
\hline
\end{tabular}

\section{Análisis Funcional}

Todas las variables fueron sometidas al análisis de varianza y para determinar las diferencias estadísticas de rangos múltiples se utilizó la prueba de Tukey al 5\% de significancia.

\section{Manejo del Ensayo}

Para el desarrollo del ensayo experimental se realizaron las siguientes labores:

\section{Análisis del Suelo}

La toma de muestras del suelo se realizó en zigzag de toda el área del terreno, las cuales fueron las primeras diez sub-muestras y luego de mezclarlas se tomó una con un peso de dos $\mathrm{kg}$. 


\title{
Preparación del Suelo
}

La preparación de suelo se realizó efectuando dos pases de rastra en ambos sentidos con la finalidad de que el suelo quede completamente mullido y así obtener buena germinación de la semilla.

\begin{abstract}
Abonado
Se aplicó abonado orgánico a base de Eco - Abonaza $(20 \mathrm{~kg} / \mathrm{ha})$ dos semanas antes de la siembra.
\end{abstract}

\section{Siembra}

La siembra se efectuó en forma manual, la distancia comprendida entre surcos fue 0,60 m y 0,30 entre planta. Se utilizó dos semillas por golpe.

\section{Fertilización}

Para la fertilización se aplicó 10-30-10 al momento de la siembra en dosis de $40 \mathrm{~kg} / \mathrm{ha}$; a los 15 días después de la siembra como abono foliar se utilizó el aminoácido Guardián en dosis de $2 \mathrm{~L} /$ ha y a los 45 días después de la siembra se aplicó 8-20-20 en dosis de $100 \mathrm{~kg} / \mathrm{ha}$.

\section{Riego}

El ensayo se lo realizó en la época seca, se regó por gravedad por canal abierto según las necesidades del cultivo.

\section{Control de Malezas}

Se efectuó la deshierba de forma manual a los 20 días de iniciado el cultivo.

\section{Control Fitosanitario}

Para el control de Alternaria sp se aplicó Mancozeb + Clorpirifos en dosis de $3,0 \mathrm{~kg}+1 \mathrm{~L} /$ ha a los 15 días después de la siembra y a los 45 días después de la siembra se utilizó Cymoxanil + Mancozeb, en dosis de 2,5 $\mathrm{kg} / \mathrm{ha}$. No se presentaron insectos debido a la aplicación preventiva del producto durante el desarrollo del cultivo.

\section{Cosecha}

La cosecha se realizó en forma manual en el área útil de la parcela experimental, cuando los granos alcanzaron la madurez fisiológica en cada parcela. Se cosechó todas las plantas que conformaron, luego se procedió a tomar los datos correspondientes.

\section{Datos evaluados}

Para estimar los efectos de los tratamientos se tomaron los siguientes datos: 


\section{Altura de la Planta}

Se obtuvo al azar 10 plantas en el área útil a los 30, 60 y 90 días después de la siembra y se procedió a la medición desde la base de la planta hasta la última hoja.

\section{Días a Floración}

Se contó los días a partir de la siembra hasta que aparecieron el 50\% de las flores en las plantas de la parcela neta de la unidad experimental.

\section{Días a Maduración}

El tiempo de maduración se registró en porcentajes a partir de los 90 días de edad del cultivo y se evaluó semanalmente hasta que las vainas presentaron la madurez (cosecha).

\section{Longitud de Vainas}

En el área útil de cada parcela se tomaron 10 vainas al azar y se midió su longitud desde la base hasta la punta de la vaina; esta medida se expresó en centímetros.

\section{Número de Vainas Por Planta}

Se contabilizó el número de vainas de diez plantas de la parcela neta, tomadas al azar, procedimiento que se realizó en cada unidad experimental, en la misma fecha de la cosecha.

\section{Número de Semillas Por Vaina}

Se contó el número de granos o semillas de las vainas de diez plantas de la parcela neta.

\section{Peso de 100 Semillas}

Se tomaron 100 granos por parcela experimental teniendo en cuenta que estuviesen libres de enfermedades y se procedió a pesar en una balanza digital, registrando su peso en $\mathrm{g}$.

\section{Rendimiento de Grano.}

Se procedió a cosechar todas las plantas de la parcela neta, se trilló manualmente y se pesó individualmente, sacando un promedio y se transformó a $\mathrm{kg} /$ parcela y posteriormente a $\mathrm{kg} / \mathrm{ha}$, para este procedimiento se utilizó una balanza.

\section{Resultados}

\section{Altura de Planta}

El análisis de varianza en los tratamientos, reportó diferencias altamente significativas en las evaluaciones efectuadas. Los promedios 
generales fueron 16,$28 ; 42,10$ y $90,91 \mathrm{~cm}$ y los coeficientes de variación son 18,$24 ; 18,23$ y $9,39 \%$, respectivamente.

En la variable altura de planta a los 30 días después de la siembra, el mayor valor lo presentó la variedad Canario con la utilización del calendario lunar, con 24,50 cm; estadísticamente igual a la variedad Yunguilla, sin calendario lunar y Paragachi, con calendario lunar y superiores estadísticamente a los demás tratamientos, obteniendo el menor valor la variedad Canario, sin calendario lunar, con $8,88 \mathrm{~cm}$.

En la evaluación de altura de planta a los 60 días, la variedad Paragachi, sin la aplicación del calendario lunar reportó el mayor valor $(54,25$ $\mathrm{cm}$ ), igual estadísticamente a Paragachi y Canario utilizando calendario lunar, y estos superiores estadísticamente a los demás tratamientos, siendo la variedad Yunguilla, sin la utilización del calendario lunar el menor valor $(32,18 \mathrm{~cm})$.

En altura de planta a los 90 días, se determinó que la variedad Paragachi, empleando calendario lunar obtuvo el mayor valor, con $87,35 \mathrm{~cm}$, superiores estadísticamente a los demás tratamientos, siendo la variedad Yunguilla sin la utilización del calendario lunar la que obtuvo el menor valor con $44,43 \mathrm{~cm}$.

Cuadro 2. Altura de planta a los 30, 60 y 90 días, utilizando el calendario agrícola lunar, en tres variedades de fréjol en el cantón Antonio Ante, provincia de Imbabura. UTB, FACIAG.

\begin{tabular}{|c|c|c|c|c|c|}
\hline \multicolumn{3}{|c|}{ Tratamientos } & \multicolumn{3}{|c|}{ Altura de planta $(\mathrm{cm})$} \\
\hline $\mathrm{N}^{\circ}$ & Variedades & Calendario Lunar & 30 días & 60 días & 90 días \\
\hline $\mathrm{T} 1$ & Paragachi & Calendario & $18,18 \mathrm{ab}$ & $49,90 \mathrm{ab}$ & 87,35 a \\
\hline $\mathrm{T} 2$ & Paragachi & Sin calendario & $14,10 b c$ & $54,25 \mathrm{a}$ & $72,78 \mathrm{~b}$ \\
\hline $\mathrm{T} 3$ & Canario & Calendario & $24,50 \mathrm{a}$ & $46,80 \mathrm{abc}$ & $66,28 \mathrm{~b}$ \\
\hline $\mathrm{T} 4$ & Canario & Sin calendario & $8,88 \mathrm{c}$ & $35,53 \mathrm{bc}$ & $48,28 \mathrm{c}$ \\
\hline T5 & Yunguilla & Calendario & $13,13 \mathrm{bc}$ & $33,98 \mathrm{bc}$ & $46,35 \mathrm{c}$ \\
\hline T6 & Yunguilla & Sin calendario & $18,88 \mathrm{ab}$ & $32,18 \mathrm{c}$ & $44,43 \mathrm{c}$ \\
\hline \multicolumn{3}{|c|}{$X$} & $\begin{array}{c}16,28 \\
* *\end{array}$ & $\begin{array}{c}42,10 \\
* *\end{array}$ & $\begin{array}{c}60,91 \\
* *\end{array}$ \\
\hline \multicolumn{3}{|c|}{ C.V. (\%) } & 18,24 & 18,23 & 9,39 \\
\hline
\end{tabular}

Promedios con una misma letra no difieren significativamente, según la Prueba de Tukey.

\section{Días a Floración}

En el Cuadro 3, se encuentran los valores promedios de días a floración. El análisis de varianza en los tratamientos, encontró diferencias altamente significativas, el promedio general fue de 54,04 días y el coeficiente de variación 2,64 \%. 
En la evaluación de días a floración, la variedad Canario, sin la aplicación del calendario lunar presentó el mayor valor (71,00 días), superior estadísticamente a los demás tratamientos, siendo la variedad Yunguilla, con la utilización del calendario lunar la que alcanzó el menor valor (43,00 días).

\section{Días a Maduración}

Los valores promedios de días a maduración, se presentan en el Cuadro 3. El análisis de varianza en los tratamientos, reportó diferencias altamente significativas. El promedio general fue de 120,63 días y el coeficiente de variación $3,26 \%$.

En la variable días a maduración, el mayor valor lo presentó la variedad Canario sin la utilización del calendario lunar, con 149,25 días, estadísticamente igual a la variedad Canario, con calendario lunar y estos superiores estadísticamente a los demás tratamientos, logrando el menor valor la variedad Yunguilla, con calendario lunar, con 94,25 días.

Cuadro 3. Días a floración y maduración, en utilizando el calendario agrícola lunar, en tres variedades de fréjol en el cantón Antonio Ante, provincia de Imbabura. UTB, FACIAG.

\begin{tabular}{|c|c|c|c|c|}
\hline \multicolumn{3}{|c|}{ Tratamientos } & \multirow{2}{*}{$\begin{array}{c}\text { Días a } \\
\text { floración }\end{array}$} & \multirow{2}{*}{$\begin{array}{c}\text { Días a } \\
\text { maduración }\end{array}$} \\
\hline $\mathrm{N}^{\mathrm{o}}$ & Variedades & Calendario Lunar & & \\
\hline $\mathrm{T} 1$ & Paragachi & Calendario & $45,00 \mathrm{de}$ & $110,75 \mathrm{c}$ \\
\hline $\mathrm{T} 2$ & Paragachi & Sin calendario & $52,00 \mathrm{c}$ & $122,00 \mathrm{~b}$ \\
\hline T3 & Canario & Calendario & $65,00 \mathrm{~b}$ & $144,50 \mathrm{a}$ \\
\hline $\mathrm{T} 4$ & Canario & Sin calendario & $71,00 \mathrm{a}$ & $149,25 \mathrm{a}$ \\
\hline T5 & Yunguilla & Calendario & $43,00 \mathrm{e}$ & $94,25 \mathrm{~d}$ \\
\hline T6 & Yunguilla & Sin calendario & $48,25 \mathrm{~d}$ & $103,00 \mathrm{~cd}$ \\
\hline \multicolumn{3}{|c|}{$\mathrm{X}$} & $\begin{array}{c}54,04 \\
* *\end{array}$ & $\begin{array}{c}120,63 \\
* *\end{array}$ \\
\hline \multicolumn{3}{|c|}{ C.V. (\%) } & 2,64 & 3,26 \\
\hline
\end{tabular}

Promedios con una misma letra no difieren significativamente, según la Prueba de Tukey.

\section{Longitud de Vainas}

El análisis de varianza en los tratamientos, obtuvo diferencias altamente significativas, el promedio general fue de $12,66 \mathrm{~cm}$ y el coeficiente de variación $8,99 \%$.

En esta variable, el mayor valor lo consiguió la variedad Canario con la utilización del calendario lunar, con 14,55 cm; estadísticamente igual a las variedades Paragachi, utilizando el calendario lunar y Yunguilla con y sin 
calendario lunar y estos superiores estadísticamente a los demás tratamientos, siendo el menor valor la variedad Canario, sin utilizar calendario lunar, con $10,20 \mathrm{~cm}$.

\section{Vainas Por Planta}

El análisis de varianza en los tratamientos, obtuvo diferencias altamente significativas. El promedio general fue de 14,71 vainas y el coeficiente de variación 10,98\%.

En la evaluación de vainas por planta, la variedad Yunguilla, con la aplicación del calendario lunar presentó el mayor valor (19,25 vainas), igual estadísticamente a la variedad Canario, utilizando el calendario lunar; Yunguilla sin emplear calendario lunar, siendo estos superiores estadísticamente a los demás tratamientos, sin embargo la variedad Canario, sin la utilización del calendario lunar alcanzó el menor valor (9,75 vainas).

Cuadro 4. Longitud de vainas y vainas por planta, utilizando el calendario agrícola lunar, en tres variedades de fréjol en el cantón Antonio Ante, provincia de Imbabura. UTB, FACIAG.

\begin{tabular}{|c|c|c|c|c|}
\hline \multicolumn{3}{|c|}{ Tratamientos } & \multirow{2}{*}{$\begin{array}{l}\text { Longitud de } \\
\text { vainas }(\mathrm{cm})\end{array}$} & \multirow{2}{*}{$\begin{array}{c}\text { Vainas por } \\
\text { planta }\end{array}$} \\
\hline $\mathrm{N}^{\mathrm{o}}$ & Variedades & Calendario Lunar & & \\
\hline $\mathrm{T} 1$ & Paragachi & Calendario & $13,63 \mathrm{ab}$ & $14,50 \mathrm{bc}$ \\
\hline $\mathrm{T} 2$ & Paragachi & Sin calendario & $11,68 b c$ & $12,00 \mathrm{~cd}$ \\
\hline $\mathrm{T} 3$ & Canario & Calendario & $14,55 \mathrm{a}$ & $15,75 \mathrm{ab}$ \\
\hline $\mathrm{T} 4$ & Canario & Sin calendario & $10,20 \mathrm{c}$ & $9,75 \mathrm{~d}$ \\
\hline $\mathrm{T} 5$ & Yunguilla & Calendario & $13,70 \mathrm{ab}$ & 19,25 a \\
\hline T6 & Yunguilla & Sin calendario & $12,20 \mathrm{abc}$ & $17,00 \mathrm{ab}$ \\
\hline \multicolumn{3}{|c|}{ X } & $\begin{array}{c}12,66 \\
* *\end{array}$ & $\begin{array}{c}14,71 \\
* *\end{array}$ \\
\hline \multicolumn{3}{|c|}{ C.V. $(\%)$} & 8,99 & 10,98 \\
\hline
\end{tabular}

Promedios con una misma letra no difieren significativamente, según la Prueba de Tukey.

\section{Semillas Por Vainas}

En el análisis de varianza en los tratamientos, se encontró diferencias altamente significativas, el promedio general fue de 5,50 semillas y el coeficiente de variación $14,85 \%$.

En la evaluación de semillas por vaina, la variedad Yunguilla, con la aplicación del calendario lunar presentó el mayor valor (7,00 semillas por vainas), estadísticamente igual a la variedad Paragachi y Canario con la 
utilización de calendario lunar; Yunguilla sin emplear calendario lunar y estos superiores estadísticamente al resto de tratamientos, reportando el menor valor la variedad Canario, sin calendario lunar (4,25 semillas por vainas).

\section{Peso de 100 Semillas}

Los valores promedios del peso de 100 semillas, se presentan en el Cuadro 5. El análisis de varianza en los tratamientos, logró diferencias altamente significativas. El promedio general fue de $55,25 \mathrm{~g}$ y el coeficiente de variación $25,09 \%$.

En la variable peso de 100 semillas, el mayor valor lo obtuvo la variedad Canario con la utilización del calendario lunar; con 73,25 g, estadísticamente igual a la variedad Paragachi, con y sin calendario lunar; Yunguilla con calendario lunar y estos superiores estadísticamente a los demás tratamientos, siendo el menor valor la variedad Canario, sin calendario lunar con $38,00 \mathrm{~g}$.

Cuadro 5. Semillas por vainas y peso de 100 semillas, utilizando el calendario agrícola lunar, en tres variedades de fréjol en el cantón Antonio Ante, provincia de Imbabura. UTB,

FACIAG.

\begin{tabular}{|c|c|c|c|c|}
\hline \multicolumn{3}{|c|}{ Tratamientos } & \multirow{2}{*}{$\begin{array}{l}\text { Semillas por } \\
\text { vainas }\end{array}$} & \multirow{2}{*}{$\begin{array}{l}\text { Peso de } 100 \\
\text { semillas }(\mathrm{g})\end{array}$} \\
\hline $\mathrm{N}^{\mathrm{o}}$ & Variedades & Calendario Lunar & & \\
\hline $\mathrm{T} 1$ & Paragachi & Calendario & $6,00 \mathrm{ab}$ & $69,00 \mathrm{ab}$ \\
\hline $\mathrm{T} 2$ & Paragachi & Sin calendario & $4,75 \mathrm{~b}$ & $44,50 \mathrm{ab}$ \\
\hline $\mathrm{T} 3$ & Canario & Calendario & $5,25 \mathrm{ab}$ & $73,25 \mathrm{a}$ \\
\hline $\mathrm{T} 4$ & Canario & Sin calendario & $4,25 \mathrm{~b}$ & $38,00 \mathrm{~b}$ \\
\hline T5 & Yunguilla & Calendario & $7,00 \mathrm{a}$ & $68,25 \mathrm{ab}$ \\
\hline T6 & Yunguilla & Sin calendario & $5,75 \mathrm{ab}$ & $38,50 \mathrm{~b}$ \\
\hline & $\begin{array}{r}\text { X } \\
\text { F. } \\
\text { C.V. }\end{array}$ & & $\begin{array}{c}5,50 \\
* * \\
14,85\end{array}$ & $\begin{array}{c}55,25 \\
* * \\
25,09\end{array}$ \\
\hline
\end{tabular}

Promedios con una misma letra no difieren significativamente, según la Prueba de Tukey.

\section{Rendimiento}

El análisis de varianza en los tratamientos, alcanzó diferencias altamente significativas. El promedio general fue de 2904,64 kg/ha y el coeficiente de variación 3,49\%.

En la variable rendimiento, el mayor valor lo presentó la variedad Canario con la utilización del calendario lunar $(3325,00 \mathrm{~kg} / \mathrm{ha})$ 
estadísticamente superior a los demás tratamientos, alcanzando el menor valor la variedad Canario, sin calendario lunar $(2703,25 \mathrm{~kg} / \mathrm{ha})$.

Cuadro 6. Rendimiento en tres variedades de fréjol, utilizando el calendario agrícola lunar, en el cantón Antonio Ante, provincia de Imbabura. UTB, FACIAG.

\begin{tabular}{|c|c|c|c|}
\hline \multicolumn{3}{|c|}{ Tratamientos } & \multirow{2}{*}{$\begin{array}{c}\text { Rendimiento } \\
(\mathrm{kg} / \mathrm{ha})\end{array}$} \\
\hline $\mathrm{N}^{\mathrm{N}}$ & Variedades & Calendario Lunar & $3025,00 \mathrm{~b}$ \\
\hline $\mathrm{T} 1$ & Paragachi & Calendario & $2933,00 \mathrm{bc}$ \\
\hline $\mathrm{T} 2$ & Paragachi & Sin calendario & $3325,00 \mathrm{a}$ \\
\hline $\mathrm{T} 3$ & Canario & Calendario & $2703,25 \mathrm{c}$ \\
\hline $\mathrm{T} 4$ & Canario & Sin calendario & $2986,50 \mathrm{~b}$ \\
\hline $\mathrm{T} 5$ & Yunguilla & Calendario & $2811,50 \mathrm{bc}$ \\
\hline T6 & Yunguilla & Sin calendario & 2964,04 \\
$* *$
\end{tabular}

Promedios con una misma letra no difieren significativamente, según la Prueba de Tukey

\section{DISCUSIÓN}

Almanaque Agropecuario (2009), sostienen que la luna creciente, adopta la forma de "C": la savia sube a la parte aérea. Se recomienda para plantar y podar en los casos que se quiera favorecer el rendimiento productivo de los árboles. La siembra de productos de desarrollo aéreo excepto aquellas que se puedan espigar o granar a causa del calor. Es la responsable de la estimulación de las plantas de gran crecimiento vegetativo, abonos verdes, lechugas, además de ser muy propicia para la fertilidad, por lo que resulta el mejor momento para sembrar todas las plantas que crecen en altura y dan frutos, como tomates, frijoles y vainitas.

Bakach (2012), afirma que en el Almanaque (Calendario lunar), se indican los días recomendados para 21 actividades agrícolas las mismas que están orientadas para lograr mejores resultados de acuerdo a la característica que exige cada actividad en concordancia con los ritmos lunares. De allí, que del oportuno manejo de los suelos se puede disminuir la incidencia de plagas que se albergan en él y mantener por un tiempo más prolongado la aireación del mismo; de una siembra oportuna logrará plantas más productivas y resistentes al ataque de plagas y enfermedades; en el trasplante logra un mayor prendimiento; con las podas mejora la calidad y cantidad de frutos sin debilitar a la planta; en los injertos se logra un mejor prendimiento; el abono o compost tiene una maduración más rápida y de calidad; el ensilaje de pastos (siega) se 
conserva por más tiempo; la madera cortada tiene mayor duración y no es atacada por insectos; las cosechas se conservan por más tiempo y su sabor, olor y textura son más adecuados; al controlar hongos e insectos se logran mejores resultados por su efectividad al realizar en los días en que son más vulnerables; al fertilizar se logra un mayor aprovechamiento de la planta con menor cantidad de fertilizante; con el riego el agua penetra y se mantiene por más tiempo en el suelo.

Infojardin (2012), menciona que estudios realizados revelan que durante las fases de cuarto creciente, luna llena, cuarto menguante y luna nueva el número de vainas por planta no se altera; el peso de las semillas es significativamente inferior al obtenido en luna nueva; la protección con tul contribuye a reducir el número de vainas por planta y el peso promedio total de las semillas y no sirvió como cubierta protectora del ataque de insectos; no se detectaron diferencias estadísticas en cuanto al daño ocasionado por insectos durante las cuatro fases lunares y entre las plantas cubierta y no cubiertas.

Murillo et al. (2007), mencionan que INIAP 428 Canario tiene las siguientes características: hábito de crecimiento: determinado; altura de planta (cm): 50 a 70; color de la flor: blanca; largo de la vaina (cm): 10 a 12; color del grano tierno: crema; color del grano seco: amarillo (canario); forma del grano: redondo; tamaño del grano seco: grande; días a floración: 65 a 80; días a la cosecha en verde: 125 a 150; día a la cosecha en seco: 150 a 165; número de vainas por planta: 11 a 16; número de granos por vaina: 4 a 5; peso de 100 granos secos (g): 42 a 45; peso de 100 granos tiernos (g): 80 a 90; adaptación: 1400 a 2400 msnm; rendimiento: grano seco 1933 a $2880 \mathrm{~kg} / \mathrm{ha}$ y vaina verde: 8627 a $11933 \mathrm{~kg} / \mathrm{ha}$. Resistente a roya; resistencia intermedia a antracnosis, ascoquita y añublo del halo. Rango de adaptación: 2400 a $2800 \mathrm{msnm}$; calidad nutricional (base seca): Proteína: 26.56\%, Fibra: 6.81\%, Calcio: $0.12 \%$, Fósforo: 0.41\%, Hierro: 23 ppm, Zinc: 36 ppm.

Olmedo (2009), aclara que los campesinos toman en cuenta la fase de la luna para realizar sus cultivos agrícolas, y cita las siguientes actividades. La preparación del terreno cuando es un sistema de roce, tumba y quema, la prácticas se realiza en cuarto menguante o creciente, principalmente para la tumba de los árboles, pues la madera obtenida dura más y es más resistente al ataque de las plagas.

Orbe (2011), expresa que Paragachi es una variedad de hábito de crecimiento arbustivo con guía; semiprecóz, tolerante a la sequia, de buen rendimiento y grano de tipo exportación. Las características morfológicas son: altura 70 a 90 cm; color de la flor: blanca; color del follaje: verde normal; largo del foliolo central: 12 a $15 \mathrm{~cm}$; ancho del foliolo central: 8 a $9 \mathrm{~cm}$; largo de la vaina: 12 a $14 \mathrm{~cm}$; forma de la vaina: recta; color vaina en madurez: amarilla; color del grano tierno: blanco/rosado; color grano seco: rojo moteado con 
crema; longitud del grano: 12 a $16 \mathrm{~mm}$; forma del grano: elongada-ovoide; tamaño del grano: grande. Las características agronómicas son: días a la floración: 45 a 50; días a la cosecha en verde: 85 a 95; días a la cosecha en seco: 95 a 110; número vainas/planta: 12 a 15; numero granos/vaina 4 a 5; peso de 100 granos: 45 g; rendimiento en grano seco: 1200 a $2000 \mathrm{~kg} / \mathrm{ha}$; altitud de cultivo: 1800 a $2400 \mathrm{msnm}$; susceptible a enfermedades foliares: roya, ceniza, pudriciones de raíz; resistente a Fusarium oxysporum; intermedia a Fusarium solani; tolerante a deficiencia de agua. Las características de calidad son en porcentaje: proteína (base seca): 21,63; fibra: 6,58; cenizas: 4,52; extracto etéreo: 2,58 y extracto libre de nitrógeno: 64,41.

Wikipedia (2012), en su web señala que un calendario lunar es la forma de calcular los años no según los ciclos del sol (calendario solar, como el calendario occidental) sino según los de la luna. En el calendario lunar, cada lunación corresponde a un "mes lunar"; es decir, a cada período comprendido entre dos momentos en que la luna se halla exactamente en la misma fase (sea esta creciente o menguante) se le llama "mes lunar". Cada mes lunar corresponde a 29,53 días solares.

La misma web indica que el "mes lunar" siempre fue utilizado por los seres humanos para calcular ciertas regularidades en la Naturaleza, como el ciclo sexual de las mujeres o las mareas. El comienzo del mes lunar es un punto arbitrario que varía según la cultura; así, por ejemplo, el calendario chino considera a la luna nueva (es decir, al primer día en que la luna no se ve en el cielo) como el comienzo del mes, mientras que otros calendarios lunares y lunisolares toman como primer día del mes a la primera luna creciente.

La mayoría de las culturas tuvieron y tienen un calendario lunisolar; es decir, calendarios que no solo tienen en cuenta los ciclos de la luna, sino también los del sol, que determinan las estaciones. Como suele haber doce meses lunares por cada año solar, a la repetición de doce lunas se la llamó año lunar. Ahora bien, como los años lunares no coinciden con los años solares, cada tanto hay un año solar con trece lunas; los calendarios lunisolares, a pesar de guiarse según los meses de la luna, añaden cuando corresponde un mes al año, que se intercala, para que el comienzo del nuevo año solar tenga doce lunas. El calendario judío, el chino y el hindú son lunisolares. En la cultura occidental cristiana, si bien el calendario es solar para la mayoría de los efectos prácticos, ciertas fechas (como la pascua) se fijan según un calendario lunisolar.

\section{Conclusiones y recomendaciones}

De acuerdo a la interpretación de los resultados experimentales, se deducen las siguientes conclusiones. 
1. La mayor altura de planta se obtuvo sembrando la variedad Paragachi, con la utilización del calendario lunar.

2. La siembra de la variedad Yunguilla, empleando el calendario lunar agrícola reportó mayor número de vainas por planta y semillas por vaina.

3. La mayor longitud de vainas $(14,55 \mathrm{~cm})$; peso de 100 semillas $(73,25$ g) y rendimiento $(3325,00 \mathrm{~kg} / \mathrm{ha})$ lo presentó la variedad Canario utilizando calendario lunar.

4. La utilización del calendario lunar agrícola influyó positivamente en el desarrollo y producción de las variedades de fréjol.

Por las conclusiones expuestas se recomienda:

1. Utilizar el calendario lunar agrícola, debido a la influencia de las fases lunares en el cultivo de frejol ( $P$. vulgaris $L$.), variedad Canario en el cantón Antonio Ante, provincia de Imbabura.

2. Realizar estudios sobre la influencia de las fases lunares en cultivos de ciclo corto.

3. Efectuar investigaciones en diferentes zonas agroecológicas, utilizando el calendario lunar agrícola para observar respuesta en el cultivo de fréjol, variedad Canario.

\section{References:}

1. Almanaque Agropecuario (2009). Influencia del calendario lunar en el rendimiento del frijol. Disponible en

2. http://agrometeorologia.inia.gob.ve/index2.php?option=com docman \&task $=$ doc view \&gid=951\&Itemid $=28$.

3. Bakach, S. (2012). Almanaque lunar para el Ecuador. Disponible en http://www.codeso.com/Calendario-Lunar/Almanaque-Lunar.html.

4. Estadísticas Agropecuarias del Ecuador (2011). Disponible en http://www.inec.gov.ec/estadisticas/.

5. Infojardin (2012). Influencia de la luna en los cultivos. Disponible en http://foroarchive.infojardin.com/hortalizas-huerto-macetas/t218231.html.

6. Olmedo, A. (2009). Influencia de las fases lunares, (menguante y luna llena) sobre la propagación vegetativa del botón de oro Tithonia diversifolia para la formación de un banco de proteína. Disponible en http://repositorio.espe.edu.ec/bitstream/21000/2600/8/T-ESPEIASA\%20I-004190.pdf.

7. Orbe, J. (2011). "Producción de fréjol Phaseolus vulgaris, variedad Paragachi, mediante tres alternativas de producción: orgánica, convencional y mixta, en la provincia de Imbabura". Disponible en

8. http://repositorio.espe.edu.ec/handle/21000/4659.

9. Murillo, et al. (2007). INIAP-428 canario Guarandeño. Variedad mejorada de frejol arbustivo para la zona de Guaranda, Chimbo y San 
Miguel de Bolívar. Disponible en http://orton.catie.ac.cr/cgibin/wxis.exe/?IsisScript=INIAP.xis \&method=post $\&$ formato $=2 \&$ canti $\mathrm{dad}=1 \&$ expresion $=\mathrm{mfn}=001538$.

10. Wikipedia (2012). El Calendario Lunar. Disponible en http://es.wikipedia.org/wiki/Calendario_lunar 